Provided for non-commercial research and education use. Not for reproduction, distribution or commercial use.

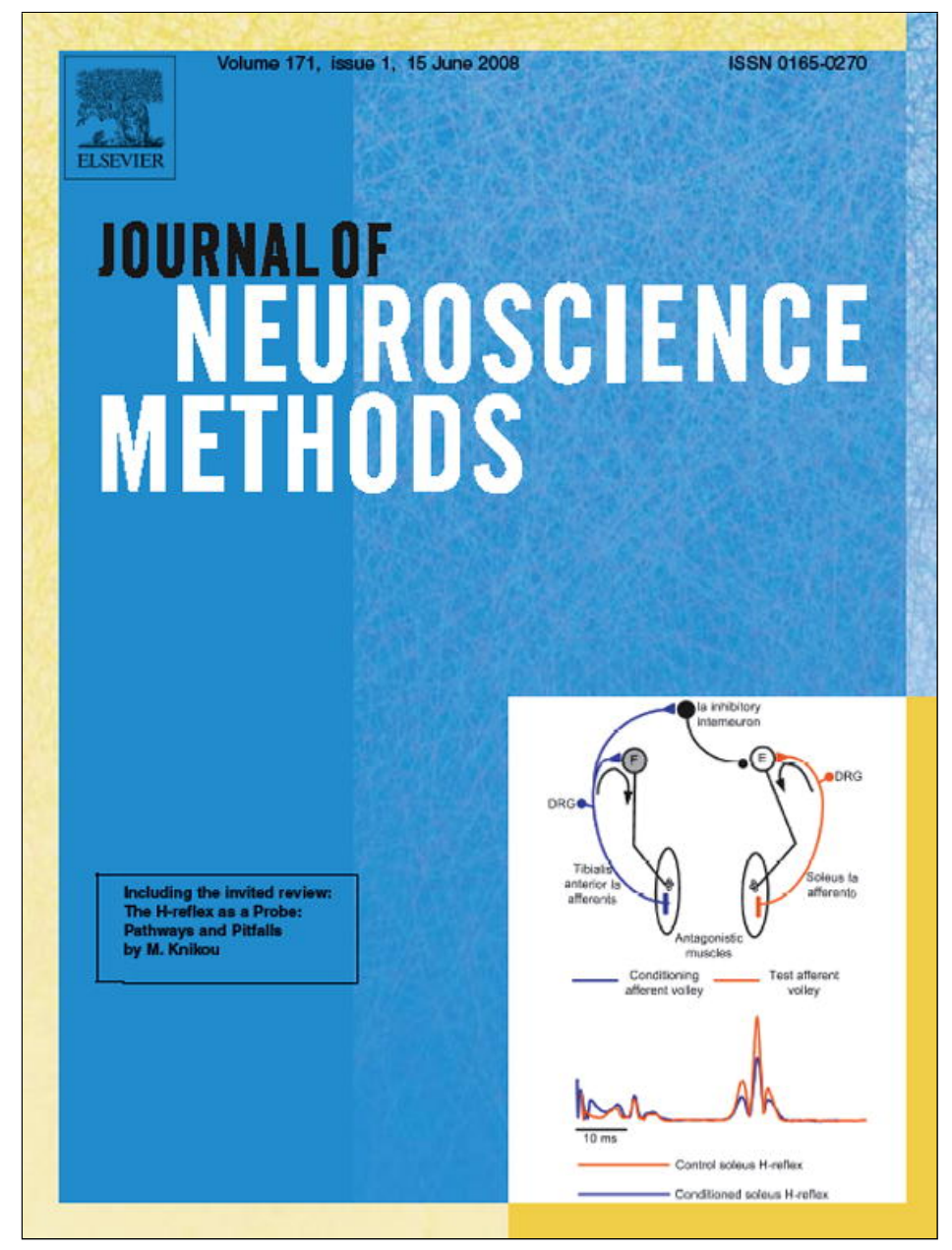

This article appeared in a journal published by Elsevier. The attached copy is furnished to the author for internal non-commercial research and education use, including for instruction at the authors institution and sharing with colleagues.

Other uses, including reproduction and distribution, or selling or licensing copies, or posting to personal, institutional or third party websites are prohibited.

In most cases authors are permitted to post their version of the article (e.g. in Word or Tex form) to their personal website or institutional repository. Authors requiring further information regarding Elsevier's archiving and manuscript policies are encouraged to visit:

http://www.elsevier.com/copyright 


\title{
Imaging honey bee brain anatomy with micro-X-ray-computed tomography
}

\author{
Willi Ribi $^{\mathrm{a}}$, Tim J. Senden ${ }^{\mathrm{b}}$, Arthur Sakellariou ${ }^{\mathrm{b}}$, Ajay Limaye $^{\mathrm{c}}$, Shaowu Zhang ${ }^{\mathrm{d}, *}$ \\ a Universität für Humanwissenschaften im Fürstentum Liechtenstein, P.O. Box 535, FL-9495 Triesen, Liechtenstein \\ ${ }^{\mathrm{b}}$ Department of Applied Mathematics, Research School of Physical Sciences and Engineering, The Australian National University, Canberra, ACT 0200, Australia \\ c Vizlab, Supercomputer Facility, The Australian National University, Canberra, ACT 0200, Australia \\ d ARC Centre of Excellence in Vision Science, Research School of Biological Sciences, The Australian National University, Canberra, ACT 2601, Australia
}

\section{A R T I C L E I N F O}

\section{Article history:}

Received 22 April 2007

Received in revised form 15 February 2008 Accepted 22 February 2008

\section{Keywords:}

X-ray-computed tomography

Honey bee brain

$3 \mathrm{D}$ reconstruction

Osmium tissue staining

\begin{abstract}
A B S T R A C T
Technologies for imaging in three dimensions are greatly desired by researchers in many biological disciplines. However, when imaging small animals such as invertebrates, the achievement of satisfactory spatial resolution and adequate contrast between tissues often requires the use of expensive and time-consuming procedures. Micro-X-ray-computed tomography $(\mu \mathrm{CT})$ is a convenient technique which is finding greater use alongside conventional microscopies. Staining with heavy metal salts, such as osmium tetroxide improves imaging in $\mu \mathrm{CT}$, and allows visualization of the 3D structure of the honey bee brain undistorted within the intact head capsule. We obtained detailed information about the morphology of the different brain compartments and were able to show their orientations, relative to each other, within the head capsule. This technique offers a significant improvement in resolution, time, and expense for the quantitative, three-dimensional analysis of developing bee brain centers. In this article, we introduce a rapid, highresolution, and inexpensive technique for the three-dimensional visualization of different compartments of the honey bee brain. A detailed discussion of the honey bee brain anatomy is provided, demonstrating that $\mu \mathrm{CT}$, with osmium staining, can indeed visualise these structures. Hence, our results show that $\mu \mathrm{CT}$ is ideally suited for researchers who are interested in the 3D visualization of small invertebrate brains.
\end{abstract}

(C) 2008 Elsevier B.V. All rights reserved.

\section{Introduction}

Honey bees are an important model animal in neurobiology. This is due to their relatively simple nervous system. In terms of structure and the number of nerve cells, the honey bee brain has approximately 1 million neurons, compared to the billion or more cells in the human brain. Nevertheless, the 'simple' brain of the honey bee is capable of an astonishing range of learning and memory phenomena and other cognitive abilities (communication, navigation, etc.). Honey bees are therefore a popular model system for analyzing neural mechanisms. Since the 17th Century, and mainly from the 19th Century, there has been extensive work on the anatomy of the honey bee brain, mainly in the form of dissections, histological investigations and reconstructions of the brain (e.g. Swammerdam, 1758; Kenyon, 1896; Cajal and Sanchez, 1915).

Histological techniques, with their broad spectrum of tissue element-specific stains and immunohistological methods, can provide useful information about molecular and cellular processes in tissues. A weakness of the histological method alone is that preparation and sectioning can damage or distort the sections, as

Abbreviations: $\mu \mathrm{CT}$, micro-computed tomography.

* Corresponding author. Tel.: +61 2 61255094; fax: +61 261253808.

E-mail address: shaowu.zhang@anu.edu.au (S. Zhang). well as render the specimen unusable for subsequent analysis by other methodologies. For decades, histologists have depended upon methods such as tried-and-true stained tissue sections (Strausfeld and Miller, 1980). Although these methods have been of enormous value in the past, how much more could be learnt if histologists could view the brain and other tissues in three dimensions? Today, these desires are being realised, thanks to greater access to computationally intensive imaging microscopies such as laser confocal, MRI, electron and X-ray tomography. Besides the qualitative advantage of a 3D perspective, this technique also provides the opportunity for quantitative image analysis. We make this distinction over 2D microscopy, for 3D microscopy allows one to analyse and study the different physical relationships of bee brain function with morpholgy. An illustration of this point is found in the techniques described in Knackstedt et al., 2006. In that work structure-function relationships were based on the direct simulation of fluid flow within the tomographic dataset of coral. As it was not possible to experimentally determine the flow within the complex structure of a coral a computational approach was fruitful. In physiology the functional aspects can be deduced by studying the changes in brain structure brought about by the learning of distinct tasks. Such research can reveal important new information about function and structure, fueling the development of new theories-in the present case, in invertebrate brain research. 
Earlier methods used to study the three-dimensional structure of the different parts of the brain relied on fixed and stained brain tissue, which was serial sectioned and 3D reconstructed (e.g. Ribi, 1975; Strausfeld, 1976; Mobbs, 1982). More recent techniques based on optical sections, acquired using confocal microscopy, give 3D impressions of certain parts of the brain (e.g. Galizia et al. 1999). It is worth contrasting the 4 principal 3D imaging techniques used today. Laser Confocal Microscopy is a very common tool, but for insect work suffers from the need for specimen transparency, appropriate fluorescent labeling of tissues and relatively anisotropic datasets (in which one data axis is much smaller than the other two). Electron Tomography also require that tissues be stained, and is limited to relatively thin specimens which, as with confocal, lead to relatively anisotropic datasets. For both these 3D microscopies, the field of view of the data may become limiting when following extended structures. $\mu$ MRI offers excellent tissue differentiation without the need for staining, as well as isotropic datasets (e.g. Haddad et al., 2004; Herberholz et al., 2004). However this technique has perhaps the most limited spatial resolution of the various microscopes, and the infrastructural cost is high. In comparison, the $\mu \mathrm{CT}$ technique provides the widest possible field of view of the microscopies mentioned, truly isotropic datasets and the possibility of imaging thick opaque specimens, at an infrastructural cost intermediate to confocal and electron tomography. In terms of resolution, $\mu \mathrm{CT}$ offers a factor of 4 improvement over $\mu \mathrm{MRI}$, whereas both confocal and Electron Tomography readily offer a factor of ten improvement over $\mu \mathrm{CT}$. Finally, it is important to note that no one technique offers the best of resolution, contrast and field-of-view all at once. Additionally, one major limitation is that if resolution could be maintained with the same field-of-view, the 3D datasets, using current computer technologies, would be prohibitively large.

Although these imaging techniques allow the nondestructive imaging of a small 3D volume, $\mu \mathrm{CT}$ methods are limited by relatively poor contrast in unstained soft tissues, such as brain tissue. To overcome this problem, a heavy metal can be used as a contrast agent to aid the imaging of the nervous system and other complex anatomical structures in small animals. Due to the tendency of heavy metal ions to differentially accumulate in most soft tissues, a higher overall signal intensity and strongly improved contrast between brain regions yields data well suited to extract 3D structure using digital post-processing techniques. Our method employs osmium tetroxide which chemically reacts with carbondouble bonds to differentially stain tissues.

\section{Materials and methods}

\subsection{Sample preparation}

Foraging worker honey bees, Apis mellifera, were immobilized by cooling to $4{ }^{\circ} \mathrm{C}$ before the head capsules were severed from the thorax, and opened from the anterior and posterior sides to allow the fixative to contact the brain directly. The brain was not removed from the head capsule, so as to maintain its original internal structure. In this way, $\mu \mathrm{CT}$ analysis preserves the natural stereo-geometry of various brain structures, unlike traditional histological techniques. The hypopharyngeal and the head salivary glands were removed, along with the head muscles. The removal of the muscle tissue is important as it stains particularly well and can interfere with the imaging of the brain tissue. The brain in the head capsule was then fixed in a mixture of $2 \%$ paraformaldehyde and $2.5 \%$ glutaraldehyde in phosphate buffer ( $\mathrm{pH} 7.2-7.4$ ) before being osmicated as for electron microscopic preparations. After dehydration with propylene oxide, the heads were embedded in Araldite (FLUKA). The excess Araldite was cut from the bee head, and the specimen was glued in an upright position on a specimen holder, made from a thin strip of Mylar film. Thus, only a thin coating of araldite covers the head.

\subsection{Micro-computed tomography facility}

X-ray-computed tomography uses a series of 2D radiographic images of a specimen to generate a 3D "tomographic" image of the structural density within the specimen. X-ray tomography uses multiple radiographic images to discern variations in 3D structural density. Each radiograph can only record a "depth-averaged" view of the specimen. Thus, any "depth" information about a particular structure is lost, as it is impossible to tell whether the structure is "in front" or "behind" any other structures from one radiograph alone. However, if a series of 2D radiographs are recorded as the specimen revolves, then with the use of an appropriate transform (e.g. Feldkamp et al., 1984), a computer can process the radiographs to generate a 3D image of the specimen. Each data point within these 3D images is called a "voxel". It is important to note that these are cubic volume elements and thus the data can be extracted in any arbitrary direction.

The $\mu \mathrm{CT}$ facility used to perform the imaging was developed in-house at The Australian National University (Sakellariou et al., 2004; Sheppard et al., 2006). It was designed to provide high quality and fidelity tomographic data for use in simulations and network modeling in porous (Knackstedt et al., 2004; Arns et al., 2005a,b) and granular materials (Aste et al., 2006). The facility can provide $<5 \mu \mathrm{m}$ resolution on samples $10 \mathrm{~mm}$ or smaller, for volumes of $\sim 8$ billion $\left(2048^{3}\right)$ voxels. The X-rays are generated via bremsstrahlung by focusing a micron-sized electron beam onto a tungsten target. Since the X-ray emission is isotropic, an area detector is used for a more efficient collection of the X-ray illumination of a specimen. Such a geometry is called a "cone-beam" and results in the voxels being cubic. One benefit of a cone-beam geometry is that the magnification of a specimen is determined simply from the proximity of the specimen to the source, compared with the distance between the detector and the source. As with many commercial $\mu \mathrm{CT}$ systems the X-ray source can be set with an acceleration voltage from $0 \mathrm{kV}$ to $225 \mathrm{kV}$, thus allowing control over the maximum energy of the Xray spectrum. For imaging small biological samples it is important to use low energies to improve contrast between tissues of different absorptivity. Additionally, the use of a metal staining technique was essential to differentiate between the fatty and non-fatty tissues within the head capsule, and necessarily limits the technique at present to non-living specimens. To maximise contrast in the bee brains, we chose to run with $40 \mathrm{kV}$ at $200 \mu \mathrm{A}$. For the bee specimens presented here, $2048^{3}$ voxels constituted unnecessarily large datasets. Instead, the detector was set to $4 \times 4$ pixel binning, so as to generate volumes with only $512^{3}$ voxels. The typical scan time was $1 \mathrm{~h}$ for 720 projections. The voxel size of $7 \mu \mathrm{m}$ in this case is not the limiting resolution; rather, the staining clarity limits the overall resolution. It was possible to scan at $1024^{3}$ giving $3.5 \mu \mathrm{m}$ voxels, but there was no discernable difference in data quality. Reconstruction into a 3D image (Sakellariou et al., 2004) and subsequent segmentation, or image binarisation (Sheppard et al., 2004) was achieved using parallel code, developed in-house, which is executed on the Australian Partnership for Advanced Computing super-computer. While our study was carried out on advanced equipment, a conventional $\mu \mathrm{CT}$ will also achieve similar results. To assist the $\mu \mathrm{CT}$ community, we have freely made available a multiplatform visualization program (see Supplementary data).

\subsection{Volume visualisation}

Once the projection data are reconstructed into a 3D image (Sakellariou et al., 2004), the first step to data exploration is 3D volume rendering. In this group, the Drishti program is used (see 
Supplementary data). It was developed in-house and allows for easy volume rendering using consumer video hardware. The main feature of this program is the interface used to extract the regions to visualise. It employs a 2D histogram, which shows a variation of the density versus the gradient of the density for the entire data set. A user has the freedom to define multiple transfer functions, plus an associated arbitrary colour map, within the histogram, thus allowing various structures to be highlighted in the 3D volume rendering.

\subsection{Quantitative investigations}

The first important step to quantitative analysis is segmentation, or image binarisation (Sheppard et al., 2004). Once the data are in this format, various tools (Knackstedt et al., 2006) are used to extract and measure the dimensions and morphology of the structures in the data set. These analysis techniques are achieved using parallel code, developed in-house and are executed on the Australian Partnership for Advanced Computing super-computer. The results from this analysis will be the topic of a subsequent paper. In this paper, we concentrate on discussing the imaging technique and on a qualitative description of the $3 \mathrm{D}$ brain images.

\section{Results}

The detailed anatomy of the honey bee brain, as visualized by the $\mu C T$ technique, is shown in the animation (see Supplementary data) and Figs. $1-3$. The voxel size of $7 \mu \mathrm{m}$ was sufficient to visualize the details of the main neuropils, such as the optic lobes, antennal lobes, the protocerebrum, the mushroom bodies with their calyces and even several of their substructures. In this frontal view (Fig. 1), the brain clearly appears laid out in the head capsule between the compound eyes on both sides, with the two antennal lobes ventrally and the median ocellus in the most dorsal part of the head. $\mu \mathrm{CT}$ thus shows the internal compartmentalization of the brain neuropil, stripped of its investing rind of cell bodies.

\subsection{Description of the neuropil of the honey bee brain}

The brain of the worker honey bee occupies a volume of around $1 \mathrm{~mm}^{3}$, and weighs about $1 \mathrm{mg}$. The total number of neurons in the brain is estimated to be $850-950,000$ (Witthöft, 1967). The principal parts of the brain are the optic lobes, the mushroom bodies, the central complex, and the antennal lobes (Figs. 1-3). The optic and

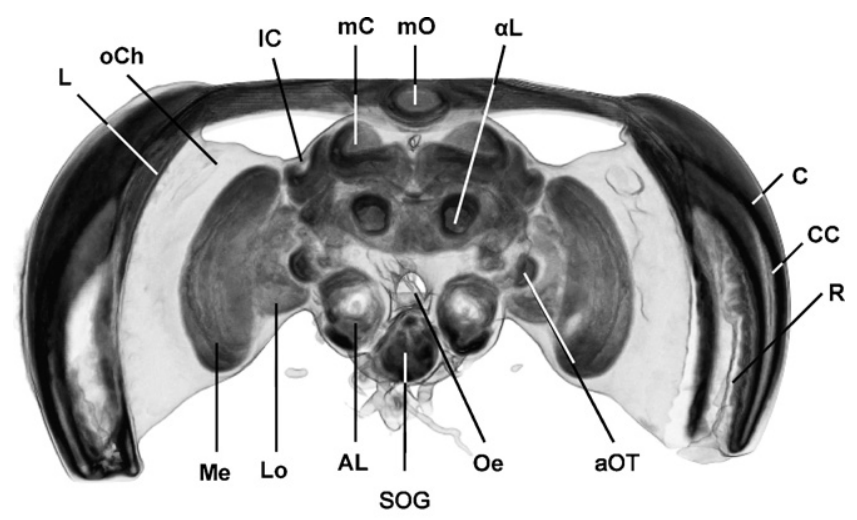

Fig. 1. X-ray $\mu \mathrm{CT}$ of the honey bee head, frontal view. The neuropil of the brain stains dark. AL: antennal lobe, aOT: anterior optic tubercle (Kenyon's optic body) C: cornea, CC: crystalline cone area, IC: left lateral calyx, $\alpha \mathrm{L}: \alpha$ lobe, L: lamina, Lo: lobula, mC: left median Calyx, Me: medulla, mO: median ocellus, oCh: outer chiasma, Oe: oesophagus, R: retina, SOG: suboesophageal ganglion. Scale bar $100 \mu \mathrm{m}$.

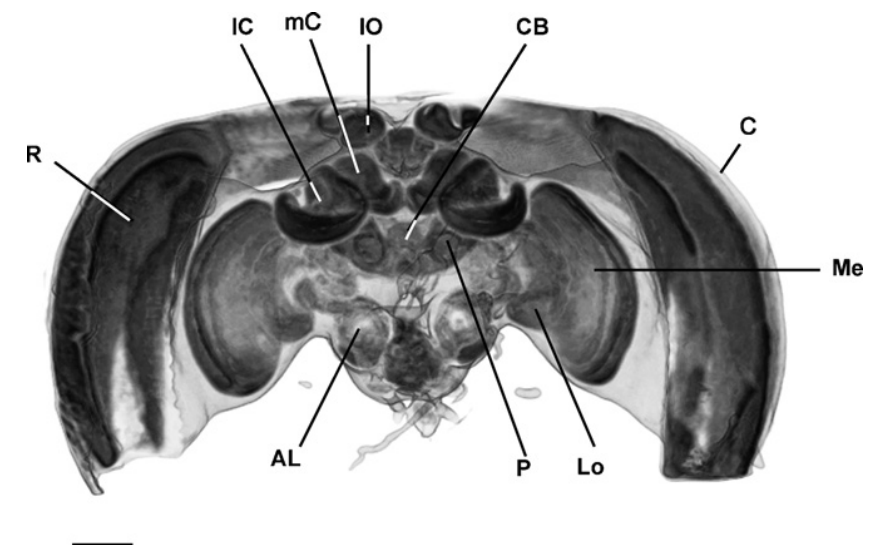

Fig. 2. X-ray $\mu \mathrm{CT}$ of the honey bee head, posterior view. AL: antennal lobe, C: cornea, CB: central body, IC: left lateral calyx, Lo: lobula, mC: left median calyx, Me: medulla, IO: left lateral ocellus, P: right pedunculus, R: retina. Scale bar $100 \mu \mathrm{m}$.

the antennal lobes are responsible for processing vision and olfaction, respectively. The mushroom bodies and the central complex constitute the most important centers for behaviour and instincts. There are various other areas of neural tissue, which surround all of these structures. However, the boundaries of these areas are not so clearly defined, and their functions are not yet well understood. Very generally, the brain consists of a layer of cells bodies, which could be thought of as a cortex, surrounding a central mass of fibers, the neuropil. Our $\mu \mathrm{CT}$ technique shows the neuropil in high contrast, while the cell body layer (perikaryon) appears unstained, and thus transparent to X-rays.

The brain or cerebrum is the principal association center of the body, receiving sensory input from the sense organs of the head and, via ascending interneurons, from the more posterior ganglia. Motor neurons to the antennal muscles are also present in the brain, but the majority of nerve cell bodies found here belong to interneurons, and the bulk of its mass is composed of their fibers. Many of the interneurons are concerned with the integration of activities; others extend down the nerve cord to the more posterior ganglia, transmitting information that controls the bee's behaviour from the brain. Three main regions are recognized in the brain, the protocerebrum, the deutocerebrum and the tritocerebrum.

The protocerebrum is bilobed, and is continuous laterally with the optic lobes. It occupies a dorsal position in the head and, as with other ganglia, is composed of a central core of nerve cell axons and their branches, surrounded by a rind of peripherally dispersed cell bodies, the perikaryon layer. A consequence of this organization is that most insect neurons are structurally unipolar. The ganglion core consists of a series of longitudinal tracts and horizontal com-

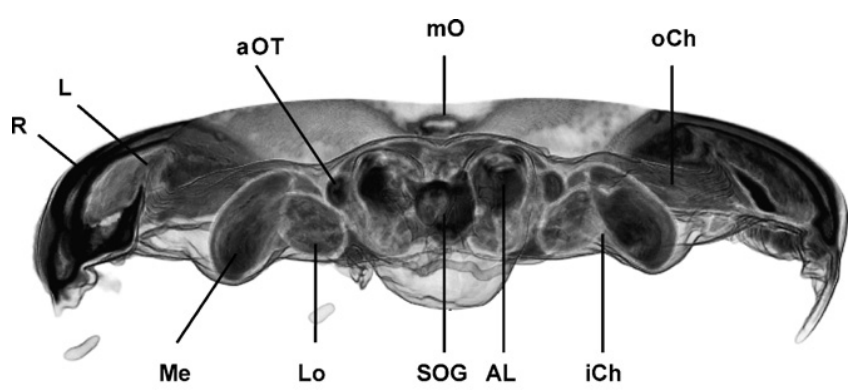

Fig. 3. X-ray $\mu \mathrm{CT}$ of the honey bee head, ventral view. AL: antennal lobe, aOT: anterior optic tubercle (Kenyon's optic body) iCh: inner chiasma, L: lamina, Lo: lobula, Me: medulla, mO: median ocellus, oCh: outer chiasma, R: retina, SOG: suboesophageal ganglion. Scale bar $100 \mu \mathrm{m}$. 
missures, representing the axonal lengths of neurons surrounded by a tangle of web branches, the neuropil.

The mushroom bodies or corpora pedunculata are a bilaterally symmetrical pair of structures deep within the brain. Each body is made up of four lobes. These are two cup-shaped structures called calyces - the median and the lateral calyx - and a stalk, the pedunculus (Figs. 1 and 2). The latter is divided into an alpha and a beta lobe. The frontal and posterior aspects show the peculiar form of the paired calyces of the honey bee. Both calyces are enlarged posteroanteriorly, and the median calyx is wider at the front than at the rear, the opposite being true of the lateral calyx (Figs. 1 and 2). The calyces comprise three concentric rings of neuropil known as the lip, collar and the basal ring. The surface of each calyx is composed of a distinct class of neurons, the so-called Kenyon cells. The alpha lobes can be seen protruding slightly from the median protocerebrum, and form a useful landmark that can be seen in the living honey bee brain (Fig. 1). Below, and to either side of the alpha lobes, are the anterior optic tubercles that represent the most anterior of the optic foci (Fig. 1) Like the four calyces and the alpha lobes, they appear darker compared to most other ganglia of the brain.

The ocelli form a fused triplet above and behind the protocerebral lobes, and contain neuropilar cores. The median ocellus pointing forward is seen in Fig. 1, whereas the two lateral ocelli are clearly visible from a posterior view (Fig. 2). The neuropils of the three ocelli arise from the posterior direction and between the median calyces. The median ocellus turns forward, emerging between the protocerebral neuropil, and is conspicuously grooved in the midline by the median furrow. Within these neuropils, the ramifications of a small number of very large interneurons and a larger group of smaller cells contact the receptor terminals. From the ocelli, these fibres form a very conspicuous ribbon over the posterior slope. Most of the large ocellar fibres terminate in the posterior neuropil, in close association with some of the compound eye optic foci and the branches of descending interneurons.

The central complex is a group of structures located in the center of the protocerebrum between the two mushroom bodies. The central complex has four main structures. These are the fan-shaped or central body, the protocerebral bridge, the ellipsoid body and the noduli. The largest of these is the fan-shaped body (Fig. 2). Each of the central complex substructures is composed of parallel strata within each substructure. This structure makes the central complex appear to possess a repetitive and modular architecture.

The protocerebral bridge connects the optic system, the antennal lobes, and other parts of the brain to the fan-shaped body. The suboesophageal bridge is the most posterior of the central complex neuropils. It can be seen at the summit of the posterior protocerebral slope. Part of the course of the tegumentary neuropil can be seen leaving trough the posterior rind to leave the brain at the margin of the lobula.

The optic lobes are lateral extensions of the protocerebrum to the compound eyes. Each consists of three neuropil masses, known as the lamina, the medulla and the lobula (Figs. 1-3). Within these masses, the arrangements of arborizations of different sets of neurons produce a layered appearance (see medulla, animation and Fig. 2). Between successive neuropils, the fibres cross over horizontally, forming the outer and inner optic chiasma, so that the neural map of the visual image is reversed and re-reversed. From the posterior aspect, the lobula, largely covered frontally by the medulla, is much more apparent (Fig. 2). Several large tracts run from the lobula over the superficial posterior surface of the protocerebrum and deutocerebrum.

The deutocerebrum contains the antennal (olfactory) lobes and the antennal mechanosensory and motor center. The latter neuropil contains the terminal arborization of mechanosensory neurons from the scape and pedicel, and perhaps from the flagellum of the antenna. Ventral and lateral to the anterior optic tubercles are two excrescences from the lateral brain, the ventro-lateral protuberances. The deutocerebral, or antennal, lobes lie tucked beneath the ventral protocerebral margin.

The antennal lobes are another prominent pair of structures located at the front of the brain (Figs. 1-3). They are regions of neuropil, one in relation to each antenna, within which are discrete balls of dense synaptic neuropil, the glomeruli. The two lobes are connected to each other across a midline by a bundle of nervous tissue, called the supraesophageal commissure. Another bundle, called the olfactorio-globularis tract, connects the antennal lobes with the mushroom bodies.

The tritocerebrum is composed of two, relatively small, bilateral lobes at the ventral base of the brain. It is a small part of the brain, and from it, the cirumoesophageal connectives pass to the suboesophageal ganglion. The two tritocerebral lobes are connected by a commissure passing behind the oesophagus.

The suboesophageal ganglion in the bee is fused with the tritocerebrum, an area of the brain that itself probably represents a primitive post-oral segment (Figs. 1 and 3). The suboesophageal ganglion lies between the main brain and the ventral cord.

\section{Discussion}

It is a well-known fact that the major steps forward in our understanding of the insect nervous system have been heralded by the development of new and increasingly powerful techniques. Here we report for the first time the use of $\mu \mathrm{CT}$ on an insect brain tissue, and give an overview of the 3D structure of the brain of the honey bee worker, Apis mellifera. We expect that this new technique will facilitate the quantitative, three-dimensional analysis of different aspects of bee brain development and architecture.

The resolution and field-of-view offered by $\mu \mathrm{CT}$ is more than sufficient for rigorous honey bee brain analysis. Given that a typical brain has a volume of $1 \mathrm{~mm}^{3}$, at a voxel resolution of $7 \mu \mathrm{m}$, a brain consists of roughly 2.9 million voxels. In measuring the effect of structure on function, variations of $10 \%$ in the volume of the brain are expected. This implies a range between $0.9 \mathrm{~mm}^{3}$ and $1.1 \mathrm{~mm}^{3}$ is expected, which relates to 2.6-3.2 million voxels, respectively. If the change of volume is assumed to be isotropic, such as a sphere, there will be a radial variation of \pm 3 voxels. Admittedly, this is verging on the limit of the sensitivity of the instrument, but it is expected that changes in brain volume will be anisotropic so the changes will be larger. Of course, with a better resolution, the variation will become larger. Nevertheless, our experience has shown that variations of 300,000 voxels are quite easily discernable and have been a routine part of other research efforts.

Micro-CT allows, within a short time, the generation of anatomical images that are not obtainable with conventional methods, thus demonstrating a new and exciting approach to invertebrate research. This tool provides a rapid screening method for anatomical features using osmium tetroxide staining and $\mu \mathrm{CT}$-based imaging. $\mu C T$-based virtual histology matches or exceeds the tissue contrast achieved by more time- and cost-intensive $\mu$ MRI, while delivering more than 4-fold higher resolution, with up to $1-3 \mu \mathrm{m}$ for $\mu \mathrm{CT}$ (incidentally, it should be noted that there are $\mu \mathrm{CT}$ instruments capable of $100 \mathrm{~nm}$ resolution, albeit with a smaller field-of-view). Most importantly, this procedure is able to provide an accurate representation of the brain in a near-natural state within the intact head capsule, with unprecedented clarity. While histology and electron microscopy provide higher spatial resolution than our images, these methods require destructive procedures, and cannot provide a natural representation of brain tissue in an undisturbed state.

MRI is currently a popular, alternative technique that also offers time savings over classical histology. However, the specialized and 
expensive equipment required for such high power magnetic resonance scans is not widely available. Furthermore, scans at useful resolutions (generally higher than are possible by $\mu \mathrm{CT}$ ) require 9-14 $\mathrm{h}$ of instrument time at exorbitant cost. We believe that the $\mu \mathrm{CT}$ technique will be most useful as a first-line screen of insect brain tissue, from which investigators could then perform traditional histological/immunohistochemical analyses of regions of interest. It could also be valuable in the high-throughput evaluation of different brain developmental stages and animal mutants. Furthermore, our specimens can be later sectioned for traditional histological light microscopy and electron microscopy investigations. The multiple uses of osmium-stained tissues will therefore speed the transition from $\mu \mathrm{CT}$-based screens to histological verification.

We expect that an entire field of $\mu$ CT-based virtual histology methods will soon emerge, given the recent advances in highresolution $\mu \mathrm{CT}$ instrumentation and the trials of existing and new electron-dense stains, such as xenon and strontium. Unlike $\mu \mathrm{MRI}$, which does not necessarily require staining, the osmium tetroxide stain for $\mu$ CT-based virtual histology is best suited to differentiate different brain compartments and structures. Because osmium staining increases with the lipid content of tissues, large differences in densities are observed between different brain centers and even within ganglia. In particular, heavy metal as a contrast agent can be used for quick and non-invasive imaging of the nervous system and other complex anatomical structures in small animals. Due to the tendency of heavy metal ions to differentially accumulate in most soft tissues, the resulting higher overall signal intensity and strongly improved contrast between structures yield data well suited for digital post-processing into three-dimensional models. We speculate that only minor modifications will be required to make our method applicable for imaging the anatomy of many other fixed invertebrates, including those of smaller size (Drosophila in prep.). However, the optimal dose of the contrast agent has to be established, the administration into smaller subjects may prove more challenging, and immobilization techniques to prevent motion artifacts during imaging must be explored. Overall, these preparations are negligible when compared to the outcome. This technique should prove invaluable to biologists interested in tracking the anatomical and morphological changes following the learning of tasks in the same animal, comparing differences between larval forms and adult forms of various ages, measuring the effects of drug application or genetically induced modifications of organs and body tissues, or simply adding to our knowledge about the anatomy of model systems used in various disciplines.

\section{Acknowledgments}

We thank Aung Si for language editing. This research was supported partly by the Australian Research Council through the ARC Centre of Excellence in Vision Science (CE0561903), and Discovery Project (DP-0450535) to S.Z.; ARC International Linkage (LX0560800) to S.Z., and W.R. T.J.S. thanks the ARC for support through his Research Fellowship and funding the construction of the $\mu \mathrm{CT}$. The Australian Partnership for Advanced Computing is gratefully acknowledged for access to the necessary computation resources.

Author contributions. W.R., and S.Z. conceived and designed the experiments. W.R., and T. S. performed the experiments. A.S., A.L. and T. S. analyzed the data. S.Z. contributed reagents/materials/analysis tools. W.R. wrote the paper.

\section{Appendix A. Supplementary data}

Supplementary data associated with this article can be found, in the online version, at doi:10.1016/j.jneumeth.2008.02.010.

\section{References}

Arns CH, Bauget F, Ghous A, Sakellariou A, Senden TJ, Sheppard AP, et al. Digital core laboratory: petrophysical analysis from 3D imaging of reservoir core fragments. Petrophysics 2005a:46:260-77.

Arns CH, Bauget F, Limaye A, Sakellariou A, Senden TJ, Sheppard AP, et al. Pore scale characterization of carbonates using micro X-ray CT. Soc Petrol Eng J 2005b;10:475-84.

Aste, T., Saadatfar, M., Senden, T.J., 2006. Local and global relations between the number of contacts and density in monodisperse sphere packs. I Stat Mech Theor Exp, Art. no. P07010.

Cajal SR, Sanchez D. Contribution al conocimiento de los centros nerviosos de los insectos. Trab Lab Invest Biol Univ Madrid 1915;13:1-168.

Feldkamp LA, Davis LC, Kress JW. Practical cone-beam algorithm. J Opt Soc Am A 1984;1:612-9.

Galizia CG, Mcllwrath SL, Menzel R. A digital three-dimensional atlas of the honeybee antennal lobe based on optical sections acquired by confocal microscope. Cell Tissue Res 1999;295(3):383-94.

Haddad D, Schaupp F, Brandt R, Manz G, Menzel R, Haase A. NMR imaging of the honeybee brain. J Insect Sci 2004;4:7.

Herberholz J, Mims CJ, Zhang X, Hu X, Edwards DH. Anatomy of a live invertebrate revealed by manganese-enhanced magnetic resonance imaging. J Exp Biol 2004:207:4543-50.

Knackstedt MA, Arns CH, Limaye A, Sakellariou A, Senden TJ, Sheppard AP, et al. Digital core laboratory: reservoir-core properties derived from 3D images. J Petrol Technol 2004;56:66-8.

Kenyon FC. The brain of the bee. A preliminary contribution to the morphology of the nervous system of the arthropoda. J Comp Neurol 1896;6:133-210.

Knackstedt MA, Arns CH, Senden TJ, Gross K. Structure and properties of clinical coralline implants measured via 3D imaging and analysis. Biomaterials 2006;27:2776-86.

Mobbs PG. The brain of the honeybee Apis mellifera. 1. The connections and spatial organizations of the musroom bodies. Philos Trans Soc Lond B 1982:298:309-54.

Ribi WA. The neurons of the first optic ganglion of the bee (Apis mellifera). Berlin/Heidelberg/New York: Springer; 1975.

Sakellariou A, Sawkins T, Senden T, Limaye A. X-ray tomography for mesoscale physics applications. Phys A Stat Mech Appl 2004;339(1-2):152-8.

Sheppard AP, Sok RM, Averdunk H. Techniques for image enhancement and segmentation of tomographic images of porous materials. Physica A 2004;339:14551.

Sheppard AP, Arns CH, Sakellariou A, Senden TJ, Sok RM, Averdunk H, et al. Quantitative properties of complex porous materials calculated from X-ray $\mu \mathrm{CT}$ images. Developments of X-ray Tomography V. In: Ulrich Bonse, editor. Proc. of SPIE, vol. 6318; 2006. p. 631811.

Strausfeld NJ. Atlas of an insect brain. Berlin/Heidelberg/New York: Springer; 1976. Strausfeld NJ, Miller TA, editors. Neuroanatomical Techniques, Insect Nervous System, vol. 1. New York/Heidelberg/Berlin: Springer; 1980.

Swammerdam J. The book of nature; 1758.

Witthöft W. Absolute Anzahl und Verteilung der Zellen im Hirn der Honigbiene. Zoomporphology 1967;61:160-84 